\title{
Uso da metodologia de superfície de resposta na otimização da extração de compostos fenólicos da casca dos frutos de Hymenaea courbaril L. (Jatobá)
}

\author{
Use of response surface methodology in optimization of \\ phenolic compounds extraction from the shell of Hymenaea \\ courbaril L. (Jatobá) fruits
}

Roger Wellington Vicente da Silva ${ }^{1}$; Glenda Monique Gonçalves Martins';

Rafael Alves do Nascimento ${ }^{2}$ (1); Alciene Ferreira da Silva Viana ${ }^{1}$;

Fagner Sousa de Aguiar ${ }^{1}$ (1); Bruno Alexandre da Silva ${ }^{3 *}$ (1)

\begin{abstract}
${ }^{1}$ Universidade Federal do Oeste do Pará (UFOPA), Instituto de Saúde Coletiva, Santarém/PA - Brasil
${ }^{2}$ Universidade Federal do Pará (UFPA), Programa de Pós-graduação em Engenharia dos Recursos Naturais da Amazônia, Belém/PA - Brasil

${ }^{3}$ Universidade Federal do Oeste do Pará (UFOPA), Laboratório de Farmacognosia, campus Tapajós, Santarém/PA

- Brasil
\end{abstract}

*Corresponding Author: Bruno Alexandre da Silva, Universidade Federal do Oeste do Pará (UFOPA), Laboratório de Farmacognosia, campus Tapajós, Rua Vera Paz, s/n, Bairro Salé, CEP: 68040-255, Santarém/PA - Brasil,

e-mail: brunoalex.ufopa@gmail.com

Cite as: Silva, R. W. V., Martins, G. M. G., Nascimento, R. A., Viana, A. F. S., Aguiar, F. S., \& Silva, B. A. (2019).

Use of response surface methodology in optimization of phenolic compounds extraction from the shell of Hymenaea courbaril L. (Jatobá) fruitsBrazilian Journal of Food Technology, 22, e2018089. https://doi.org/10.1590/19816723.08918

\begin{abstract}
Resumo
Os hábitos alimentares e medicinais dos povos da região Amazônica são ricos em frutos considerados exóticos ou subutilizados comercialmente, alguns dos quais geram considerável quantidade de resíduos. As cascas do fruto de Hymenaea courbaril L. (jatobá) foram submetidas a um processo de extração simultânea de compostos fenólicos, otimizado pela metodologia de superfície de resposta. Experimentos preliminares, investigando relação amostra-solvente $(\mathrm{g} / \mathrm{mL})$, tempo (minutos) e temperatura $\left({ }^{\circ} \mathrm{C}\right)$ de extração, indicaram os limites para o delineamento da região experimental, a qual foi monitorada por um planejamento composto central rotacional com 19 ensaios. Os resultados experimentais ajustaram-se ao modelo polinomial de segunda ordem, o qual foi capaz de descrever de forma significativa a variabilidade observada para as variáveis de resposta. As condições otimizadas foram $60,6 \%$ de etanol, $64,18^{\circ} \mathrm{C}$ e tempo de extração de $71,93 \mathrm{~min}$, e o modelo apresentou como valores preditos $35,265 \pm 2,22 \mathrm{mg}$ equivalentes ao ácido gálico/g de matéria seca e 1,210 $\pm 0,16 \mathrm{mg}$ equivalentes a rutina/g de matéria seca, para compostos fenólicos totais e flavonoides totais, respectivamente. Os valores observados experimentalmente, 33,234 $\pm 1,66 \mathrm{mg}$ equivalentes ao ácido gálico/g de matéria seca e 1,216 $\pm 0,008 \mathrm{mg}$ equivalentes a rutina/g matéria seca, para compostos fenólicos totais e flavonoides totais, respectivamente, não apresentaram diferença estatisticamente significativa em relação aos valores preditos pelo modelo, dentro de um intervalo de confiança de $95 \%$. Estes
\end{abstract}


resultados ratificaram a capacidade preditiva e a adequação da metodologia de superfície de resposta na otimização da extração simultânea para compostos fenólicos totais e flavonoides totais da casca dos frutos de jatobá.

Palavras-chave: Planejamento experimental; Polifenol; Flavonoide; Ácido gálico; Rutina; Resíduo vegetal.

\begin{abstract}
The food and medicinal habits of Amazon people are rich in fruits considered as exotic or commercially underutilized, some of which generate a considerable amount of residue. The Hymenaea courbaril L. (Jatobá) fruit shell was submitted to a simultaneous extraction of total phenolic compounds, optimized by response surface methodology. Preliminary experiments, investigating sample-solvent ratio $(\mathrm{g} / \mathrm{mL})$, time (minutes) and extraction temperature $\left({ }^{\circ} \mathrm{C}\right)$, indicate the limits for experimental region design, which was monitored by rotational central composite design consisting of 19 runs. The experimental results fitted well to the second order polynomial model generated, which was able to significantly describe the observed variability for response variables. The optimized conditions were $60.6 \%$ ethanol, $64.18{ }^{\circ} \mathrm{C}, 71.93$ minutes and the model presented predicted values of $35.265 \pm 2.22 \mathrm{mg}$ gallic acid equivalent / $\mathrm{g}$ dry matter and $1.210 \pm 0.16 \mathrm{mg}$ rutin equivalent / $\mathrm{g}$ dry matter, for total phenolic compounds and total flavonoids, respectively. The experimental observed values, $33.234 \pm 1.66 \mathrm{mg}$ gallic acid equivalent / $\mathrm{g}$ dry mater and $1.216 \pm 0.008 \mathrm{mg}$ rutin equivalent / $\mathrm{g}$ dry matter, for total phenolic compounds and total flavonoids respectively, agreed with those predicted within a $95 \%$ confidence interval. These results confirm the predictive capacity and the adequacy of response surface methodology in optimizing simultaneous extraction of total phenolic compounds and total flavonoids from shell of jatobá fruits.
\end{abstract}

Keywords: Experimental design; Polyphenol; Flavonoid; Gallic acid; Rutin; Vegetal residue.

\title{
1 Introdução
}

A comunidade científica tem direcionado atenção para as propriedades nutricionais, tecnológicas e bioativas de resíduos originados das cadeias produtivas de frutos de elevado potencial comercial (Cavalcanti et al., 2010; Infante et al., 2013), no entanto, os hábitos alimentares e medicinais dos povos da região Amazônica são ricos em frutos considerados como exóticos ou subutilizados comercialmente, alguns dos quais geram considerável quantidade de resíduos ainda pouco explorados dos pontos de vista nutricional, tecnológico e medicinal (Aquino et al., 2010; Ishimoto et al., 2007; Uchoa et al., 2008).

Hymenaea courbaril L., popularmente conhecido como Jatobá, é um vegetal de porte alto pertencente à família Fabaceae e é comumente encontrado na região tropical do Brasil (Nogueira et al., 2001; Campos \& Uchida, 2002). A parte comestível dos frutos do jatobá tem aspecto farináceo e elevados teores de proteínas e de fibras (Matos et al., 2009), apresentando, também, propriedades antioxidantes naturais e outras atividades biológicas (Lorenzi \& Matos, 2002). O súber de H. courbaril L. também apresenta propriedades medicinais, como atividades analgésicas e anti-inflamatória (Neves et al., 1993). As sementes do jatobá contêm polissacarídeos com aplicação tecnológica farmacêutica, cosmética e alimentícia (Fernandes et al., 2015). A casca do fruto do jatobá é considerada resíduo e há poucos dados disponíveis sobre sua composição química e suas propriedades nutricionais, tecnológicas e medicinais.

Atualmente, a correlação positiva entre a diminuição da incidência de doenças crônicas degenerativas, como a neoplasia maligna, e o aumento do consumo de alimentos de origem vegetal tem sido evidenciada e, dentre as propriedades bioativas relacionadas a estes alimentos, se destaca a capacidade antioxidante de compostos presentes em frutos, folhas e outras partes comestíveis do vegetal, a qual, geralmente é atribuída aos compostos fenólicos (Patil et al., 2009; Gordon et al., 2011; Gironés-Vilaplana et al., 2014; Nimalaratne $\& \mathrm{Wu}, 2015)$. 
Dentre os compostos fenólicos, os flavonoides assumem grande relevância devido à gama de atividades biológicas relacionadas aos diversos membros do grupo, tanto os heterosídeos, quanto as agliconas (Dornas et al., 2007; Machado et al., 2008).

As metodologias empregadas na extração de compostos bioativos geralmente envolvem processos com muitas etapas e com variáveis fixas, além de solventes tóxicos e gastos consideráveis de energia, fatores que inviabilizam o uso deste tipo de tecnologia em pequena escala e não garante o emprego racional da tecnologia em escala industrial, de forma economicamente viável e ecologicamente sustentável. Neste sentido, a metodologia de superfície de resposta é uma ferramenta estatística poderosa na otimização de processos extrativos para um ou vários compostos bioativos simultaneamente, pois o método prediz as condições ideais a serem empregadas durante o processo de extração desses compostos de interesse (Khuri \& Cornell, 1996; Montgomery \& Runger, 2003).

Este estudo tem por objetivo otimizar a extração de compostos fenólicos totais (CFT) e flavonoides totais (FLAT), tanto de forma isolada quanto simultaneamente, da casca do fruto do jatobá, usando a metodologia de superfície de resposta (MSR) para determinar as condições experimentais de concentração de etanol, tempo e temperatura de extração capazes de maximizar a eficiência do processo, identificando, também, qual a influência individual de cada variável experimental e as influências de suas interações no processo extrativo.

\section{Material e métodos}

\subsection{Obtenção e processamento da amostra}

Os frutos do jatobá foram coletados na região da Floresta Nacional do Tapajós (Belterra- PA), higienizados, manualmente processados, embalados em sacos plásticos e armazenadas à $-18^{\circ} \mathrm{C}$. A casca do fruto foi moída em moinho analítico e o material resultante, um pó fino e seco, descartou a necessidade de caracterização granulométrica; em seguida, o material foi armazenado ao abrigo da luz, da umidade e sob refrigeração até a execução dos ensaios. Estudos anteriores têm mostrado que os melhores rendimentos na extração de polifenóis totais são alcançados em ensaios utilizando partículas pequenas, provavelmente devido ao aumento da superfície de contato, o que ajuda a reduzir o tempo de extração (Escribano-Bailon \& Santos-Buelga, 2003; Mafart \& Béliard, 1992; Jerez et al., 2006).

Amostras do exemplar de H. courbaril L. foram comparadas com exsicatas autênticas existentes no herbário do Instituto Nacional de Pesquisas da Amazônia - INPA, depositadas sob o registro de n. ${ }^{\circ} 40.946$ $\mathrm{e}$, no procedimento de amostragem, foram considerados os frutos, maduros, inteiros, visualmente sadios e sem evidências de predação ou outro tipo de dano.

\subsection{Quantificação de compostos fenólicos totais}

O teor de compostos fenólicos totais foi determinado em espectrofotômetro UV-VIS pelo método colorimétrico de Folin-Ciocalteau. Foram transferidos $500 \mu \mathrm{L}$ dos extratos da casca de H. courbaril L. na concentração $2,5 \mathrm{mg} / \mathrm{mL}$ para tubos de vidro, sendo adicionados $2,5 \mathrm{~mL}$ de solução de reagente de Folin-Ciocalteau $1 \mathrm{~N}$ :água $(10: 90 \mathrm{v} / \mathrm{v})$. A mistura permaneceu em repouso por 5 minutos e em seguida foram adicionados $2,0 \mathrm{~mL}$ de solução de carbonato de sódio a $4 \%(\mathrm{~m} / \mathrm{v})$. Os tubos foram deixados em repouso por 2 horas ao abrigo da luz. A amostra em branco usando água destilada foi preparada nas mesmas condições. O cálculo dos resultados foi baseado em uma curva analítica de sete pontos $\left(\mathrm{R}^{2}=0,99\right)$ construída com soluções de padrão de referência de ácido gálico em concentrações de $10 \mu \mathrm{g} / \mathrm{mL}$ a $80 \mu \mathrm{g} / \mathrm{mL}$, sendo o resultado expresso em miligramas equivalentes ao ácido gálico (EAG) por grama de matéria seca. As análises foram feitas em triplicata e as absorbâncias foram medidas em $740 \mathrm{~nm}$ (Singleton \& Rossi, 1965). 


\subsection{Quantificação de flavonoides totais}

O teor de flavonoides totais foi determinado por método colorimétrico em espectrofotômetro UV-VIS. Foram transferidos $2 \mathrm{~mL}$ dos extratos de casca de $H$. courbaril L. na concentração $50 \mathrm{mg} / \mathrm{mL}$ para tubos de vidro, foi adicionado $1 \mathrm{~mL}$ de solução cloreto de alumínio $5 \%$ e, em seguida, $2 \mathrm{~mL}$ de metanol em cada tubo. A mistura reacional permaneceu em repouso por 30 minutos ao abrigo da luz. A amostra em branco usando água destilada foi preparada nas mesmas condições. O cálculo dos resultados foi baseado em uma curva analítica de sete pontos $\left(\mathrm{R}^{2}=0,99\right)$ construída com soluções de padrão de referência de rutina em concentrações de $5 \mu \mathrm{g} / \mathrm{mL}$ a $80 \mu \mathrm{g} / \mathrm{mL}$ e o resultado foi expresso em miligramas equivalentes a rutina (ERT) por grama de matéria seca. As análises foram feitas em triplicata e as absorbâncias foram medidas em $415 \mathrm{~nm}$ (Lamaison \& Carnet, 1990).

\subsection{Preparação dos extratos}

Optou-se pelo uso apenas de água e etanol como líquidos extratores no intuito de proporcionar o aproveitamento futuro da metodologia desenvolvida neste estudo em preparações de extratos que poderão ser usados em formulações alimentícias, cosméticas e farmacêuticas de uso interno e externo, com baixo risco de toxicidade para o homem e para animais, e baixo risco de contaminação do ambiente, sendo que, as concentrações ideais da solução hidroalcoólica foram determinadas pela metodologia de superfície de resposta.

\subsection{Seleção das variáveis relevantes para a extração de compostos fenólicos}

Experimentos preliminares foram realizados para avaliar os fatores relevantes na extração de compostos fenólicos a fim de delinear a região experimental para a investigação pela metodologia de superfície de resposta. Foi investigada a influência da relação amostra-solvente na extração desses compostos, considerando as proporções 1:10, 1:20, 1:40: 1:80 e 1:100 (m:v), isto é, $5 \mathrm{~g}, 2,5 \mathrm{~g}, 1,25 \mathrm{~g}, 0,625 \mathrm{~g}$ e 0,5 g de amostra foram submetidos à extração em um volume fixo de $50 \mathrm{ml}$ de uma solução de EtOH: $\mathrm{H}_{2} \mathrm{O}$ (50:50, v:v), durante 60 min e a $65^{\circ} \mathrm{C}$.

Também foi avaliada a influência do tempo de extração dos compostos de interesse nas temperaturas de $25{ }^{\circ} \mathrm{C}$ e $65^{\circ} \mathrm{C}$ em que $2,5 \mathrm{~g}$ de amostra foram submetidos à extração em um volume fixo de $50 \mathrm{~mL}$ de uma solução de EtOH: $\mathrm{H}_{2} \mathrm{O}$ (50:50, v:v), em intervalos de 20 minutos, a partir do tempo de extração 10 minutos. Após todos as extrações, se procedeu à filtração a vácuo de cada um dos extratos utilizando papel de filtro qualitativo.

Os parâmetros concentração de etanol, tempo e temperatura foram fixados em $50 \%$, 60 minutos e $65^{\circ} \mathrm{C}$ respectivamente, nos ensaios preliminares, tendo como diretriz o desenvolvimento de uma tecnologia extrativa rápida e com gasto mínimo de energia e de solvente orgânico, e em uma temperatura inferior à temperatura de degradação de alguns compostos fenólicos, a partir de $70{ }^{\circ} \mathrm{C}$ (Machado et al., 2008).

A verificação das diferenças estatísticas entre as condições experimentais foi avaliada pelo teste $t$ de Student.

\subsection{Planejamento experimental utilizando a metodologia de superfície de resposta}

O planejamento composto central consiste no desenvolvimento de um modelo preditivo elaborado pelo software Statistica ${ }^{\circledR} 10$, no qual foram definidas as variáveis independentes: temperatura $\left(\mathrm{T}^{\circ} \mathrm{C}\right)$, concentração de etanol (\%) e tempo ( $\mathrm{min})$.

O processo de extração de compostos fenólicos da casca do fruto de $H$. courbaril L. foi submetido a um planejamento composto central, no intuito tanto de otimizá-lo quanto de fornecer informações a respeito da 
influência que cada uma das variáveis independentes exerce sobre o processo isoladamente e da influência de suas interações. As condições de extração são exibidas na Tabela 1, que mostra os níveis codificados $\alpha$ e $\alpha$, como pontos extremos aos quais o processo de extração é submetido (pontos axiais), -1 e +1 como os pontos mínimos e máximos, respectivamente, definidos para o processo experimental, e 0 representando o ponto central das condições de extração.

Tabela 1. Dados das Variáveis independentes.

\begin{tabular}{|c|c|c|c|c|c|c|c|}
\hline \multirow{2}{*}{ Variáveis de independentes } & \multirow{2}{*}{ Unidade } & \multirow{2}{*}{ Notação } & \multicolumn{5}{|c|}{ Níveis } \\
\hline & & & $-\alpha$ & -1 & 0 & +1 & $\alpha$ \\
\hline Temperatura de extração & ${ }^{\circ} \mathrm{C}$ & $\mathrm{X} 1$ & 24,4 & 32 & 44 & 56 & 63,6 \\
\hline Concentração de etanol & $\%$ & $\mathrm{X} 2$ & 14,2 & 30 & 55 & 80 & 95,8 \\
\hline Tempo de extração & $\min$ & $\mathrm{X} 3$ & 13,9 & 25 & 42,5 & 60 & 71,1 \\
\hline
\end{tabular}

Foram realizados 19 ensaios, em um bloco, com cinco repetições no ponto central, de acordo com a matriz de planejamento composto central de ordem padronizada apresentada na Tabela 2. Aproximadamente $1 \mathrm{~g}$ de casca do fruto de $H$. courbaril L. pulverizada foi pesado em 19 tubos de ensaio; em seguida, adicionaram-se $20 \mathrm{~mL}$ de soluções etanol:água em concentrações variadas, de acordo com o planejamento experimental. As amostras foram levadas ao banho-maria a temperaturas e por tempos estabelecidos no planejamento experimental. Após o término dos ensaios de extração, os extratos brutos foram cuidadosamente retirados dos tubos de ensaio, submetidos à centrifugação para a separação de partículas sólidas, filtrados, envazados em frascos âmbar e identificados de acordo com as condições experimentais às quais foram submetidos. A determinação das condições experimentais otimizadas para as variáveis de resposta do experimento foi realizada com auxílio do software Statistica ${ }^{\circledR} 10$.

Tabela 2. Matriz de planejamento composto central para o experimento de extração simultânea de compostos fenólicos da casca do fruto de $H$. courbaril L.

\begin{tabular}{|c|c|c|c|c|c|}
\hline \multirow[b]{2}{*}{$\begin{array}{l}\text { Ordem de } \\
\text { ensaio }\end{array}$} & \multicolumn{3}{|c|}{ Variáveis independentes } & \multicolumn{2}{|c|}{ Variáveis resposta } \\
\hline & $\left(\mathrm{X}_{1}\right) \mathrm{T}^{\circ} \mathrm{C}$ & $\left(\mathrm{X}_{2}\right)$ ЕtOH \% & $\left(X_{3}\right) t$ min & $\begin{array}{c}\text { CFT } \\
\text { (mg EAG/g MS) }\end{array}$ & $\begin{array}{c}\text { FLAT } \\
(\mathrm{mg} \mathrm{ERT} / \mathrm{g} \text { MS) }\end{array}$ \\
\hline 1 & $(-1) 32$ & $(-1) 30$ & $(-1) 25$ & 23,244 & 0,714 \\
\hline 2 & $(-1) 32$ & $(-1) 30$ & (1) 60 & 25,469 & 0,787 \\
\hline 3 & $(-1) 32$ & (1) 80 & $(-1) 25$ & 16,289 & 0,700 \\
\hline 4 & $(-1) 32$ & (1) 80 & (1) 60 & 20,184 & 0,694 \\
\hline 5 & (1) 56 & $(-1) 30$ & $(-1) 25$ & 26,860 & 0,816 \\
\hline 6 & (1) 56 & $(-1) 30$ & (1) 60 & 31,033 & 1,062 \\
\hline 7 & (1) 56 & (1) 80 & $(-1) 25$ & 20,740 & 0,671 \\
\hline 8 & (1) 56 & (1) 80 & (1) 60 & 25,748 & 0,816 \\
\hline 9 & $(-\alpha) 24,4$ & (0) 55 & (0) 42,5 & 21,853 & 1,004 \\
\hline 10 & $(\alpha) 63,6$ & (0) 55 & (0) 42,5 & 32,424 & 1,206 \\
\hline 11 & (0) 44 & $(-\alpha) 14,2$ & (0) 42,5 & 23,522 & 0,714 \\
\hline 12 & (0) 44 & 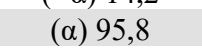 & (0) 42,5 & 16,568 & 0,497 \\
\hline 13 & (0) 44 & (0) 55 & (a) 71,1 & 21,853 & 0,801 \\
\hline 14 & (0) 44 & (0) 55 & $(-\alpha) 14,2$ & 26,860 & 0,975 \\
\hline 15 & (0) 44 & (0) 55 & (0) 42,5 & 23,800 & 0,931 \\
\hline 16 & (0) 44 & (0) 55 & (0) 42,5 & 23,522 & 0,913 \\
\hline 17 & (0) 44 & (0) 55 & (0) 42,5 & 24,078 & 0,947 \\
\hline 18 & (0) 44 & (0) 55 & (0) 42,5 & 24,357 & 0,995 \\
\hline 19 & (0) 44 & (0) 55 & (0) 42,5 & 24,913 & 0,998 \\
\hline
\end{tabular}

$\mathrm{X}_{1}$ : a temperatura de extração; $\mathrm{X}_{2}$ : concentração de etanol; $\mathrm{X}_{3}$ : tempo de extração; CFT: compostos fenólicos totais; FLAT: flavonoides totais.

O desenho experimental foi adequado ao seguinte modelo polinomial de segunda ordem apresentado na Equação 1. 
$Y=\beta_{0}+\beta_{1} X_{1}+\beta_{2} X_{2}+\beta_{3} X_{3}+\beta_{11} X_{1}^{2}+\beta_{22} X_{2}^{2}+\beta_{33} X_{3}^{2}+\beta_{12} X_{1} X_{2}+\beta_{13} X_{1} X_{3}+\beta_{23} X_{2} X_{3}$

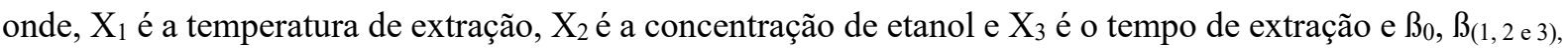
$\beta_{(11,22}$ e 33), e $\beta_{(12,13}$ e 23) são os coeficientes de regressão para o intercepto, comportamento linear, comportamento quadrático e para o comportamento de interação entre os termos, respectivamente.

A relação amostra-solvente foi mantida constante, de acordo com os resultados do experimento preliminar em 1:20 $(\mathrm{g} / \mathrm{mL})$ e as variáveis dependentes para o experimento são as respostas para CFT e FLAT.

\subsection{Determinação das condições ótimas e validação do modelo}

Usando o software Statistica ${ }^{\circledR}$ 10, foi feita a análise de desejabilidade global para as variáveis de resposta CFT e FLAT, buscando obter os rendimentos máximos. A partir das condições de concentração de etanol, temperatura e tempo de extração preditas pelo modelo como ótimas, foi realizado um experimento de validação para comprovar se o modelo preditivo de extração era adequado e o quanto as respostas preditas pelo modelo se aproximavam das respostas observadas experimentalmente.

\section{Resultados e discussão}

\subsection{Determinação das variáveis relevantes na extração de compostos fenólicos}

\subsubsection{Relação amostra-solvente}

A influência da relação amostra-solvente na extração de compostos fenólicos totais e flavonoides totais é ilustrada na Figura 1. Para a resposta FLAT, observou-se que a relação 1:10 foi a menos eficiente no processo extrativo, apresentando diferenças estatisticamente significativas em relação a todas as outras proporções estudadas; já as proporções 1:20, 1:40 e 1:80 não apresentaram diferenças estatisticamente significativas entre si e a proporção amostra-solvente 1:100, mesmo apresentando a maior eficiência em valores absolutos, diferiu estatisticamente apenas das proporções 1:10 e 1:20.

Para a resposta CFT, observou-se que a relação 1:10 também foi a menos eficiente no processo extrativo, apresentando diferenças estatisticamente significativas em relação a todas as outras proporções estudadas; já as proporções 1:20, 1:40, 1:80 e 1:100 não apresentaram diferenças estatisticamente significativas entre si (Figura 1). Diante destes resultados, optou-se por selecionar a proporção amostra-solvente 1:20 no experimento de otimização da extração simultânea pela metodologia de superfície de resposta.
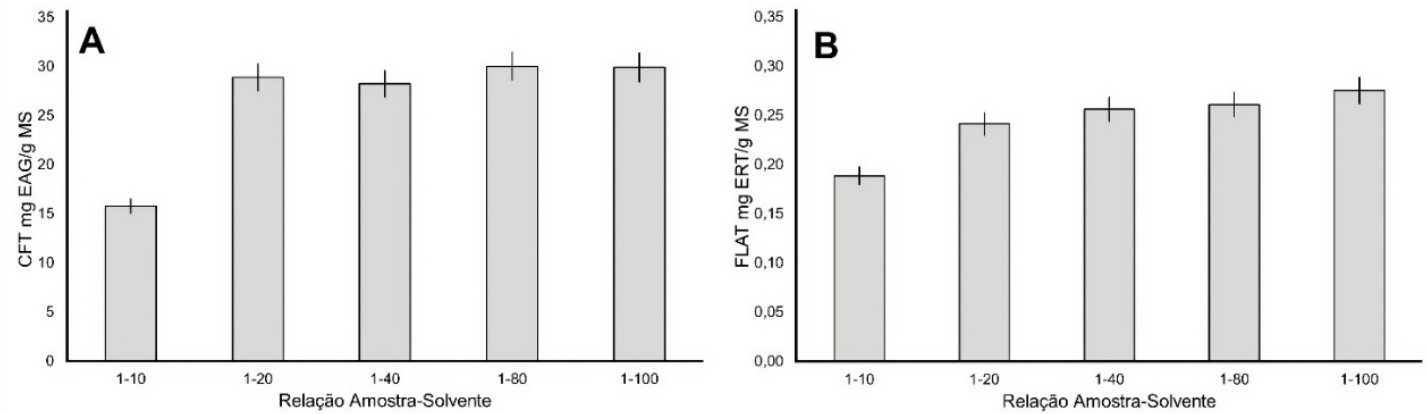

Figura 1. Efeito da relação amostra-solvente na extração de CFT (A) e FLAT (B), da casca do fruto de H. courbaril L. Barras verticais representam intervalo de confiança $(p<0,05)$. 


\subsubsection{Influência do tempo e da temperatura de extração}

Como ilustra a Figura 2, a influência da temperatura na extração de compostos fenólicos totais e flavonoides totais só se mostra estatisticamente significativa a partir de 50 e 90 minutos, respectivamente, com a melhor eficiência extrativa na temperatura de $65^{\circ} \mathrm{C}$. A influência do tempo de extração não é estatisticamente significativa a partir de 50 minutos a $25^{\circ} \mathrm{C}$ e a partir de 70 minutos a $65^{\circ} \mathrm{C}$ para compostos fenólicos totais; no entanto, a eficiência do processo extrativo para flavonoides apresentou incremento em tendência linear em relação ao tempo, tanto a $25^{\circ} \mathrm{C}$ quanto a $65^{\circ} \mathrm{C}$, sendo esta mais efetiva a $65^{\circ} \mathrm{C}$. A partir desses resultados, optou-se por limitar o experimento a temperaturas inferiores a $70{ }^{\circ} \mathrm{C}$ e observou-se que não se justifica o emprego de metodologias extrativas muito demoradas, no intuito de evitar a degradação térmica dos compostos presentes na amostra. A partir destes dados preliminares, foram então definidos os níveis mínimos e máximos das variáveis: concentração de solvente orgânico, temperatura e tempo de extração para o planejamento composto central, como já descrito na Tabela 1 (Escribano-Bailon \& Santos-Buelga, 2003).
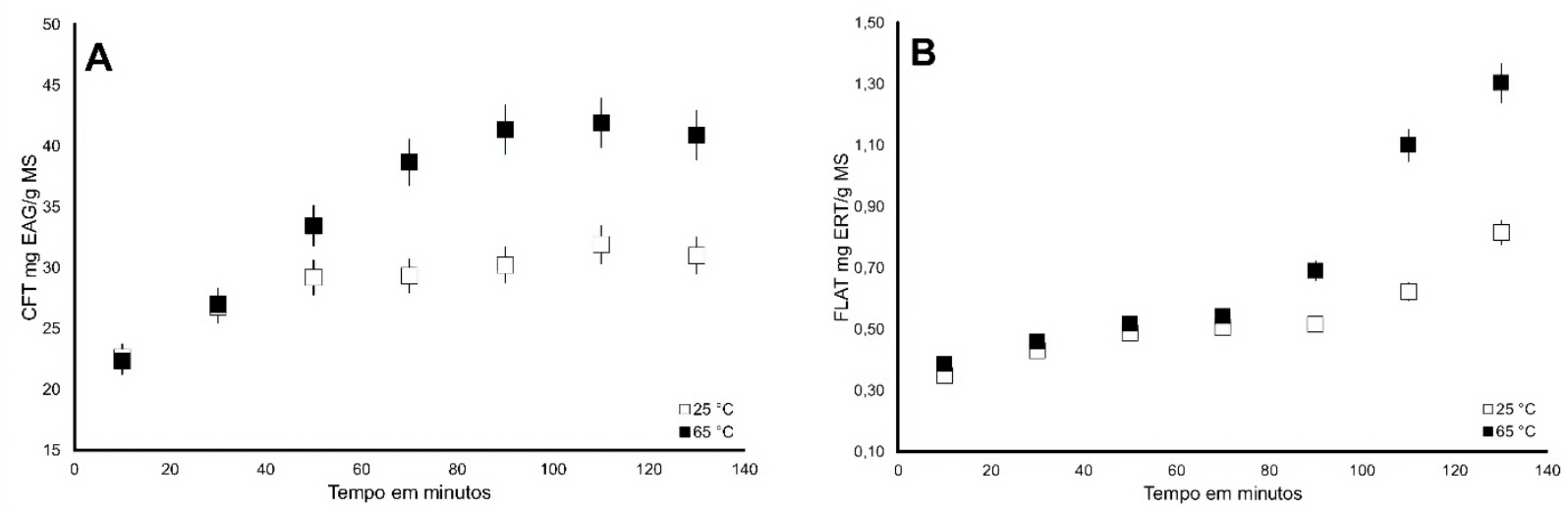

Figura 2. Efeito da influência do tempo e da temperatura na extração de CFT (A) e FLAT (B), da casca do fruto de H. courbaril L. Barras verticais representam intervalo de confiança $(p<0,05)$.

\subsection{Otimização da extração de compostos fenólicos utilizando a Metodologia da Superfície de Resposta}

\subsubsection{Adequação e significância estatística do modelo}

Os resultados para as respostas CFT e FLAT apresentados na Tabela 2 foram submetidos a analises de regressão baseadas no modelo matemático descrito na Equação 1. Os coeficientes de determinação alcançados, $\mathrm{R}^{2}=97,84 \%$ para CFT e $\mathrm{R}^{2}=95,09 \%$ para FLAT, demonstram um bom ajuste do modelo aos dados, indicando que o modelo é capaz de explicar grande parte da variabilidade apresentada pelas respostas. Os dados da análise de variância (ANOVA) demonstram a significância estatística do modelo na explicação das variações experimentais tanto para CFT quanto para FLAT em relação à resposta média, e os valores da estatística F calculada (Tabela 3) descartam a falta de ajuste do modelo em um nível de confiança de $95 \%$.

Tabela 3. ANOVA para o teste de falta de ajuste para compostos fenólicos totais (CFT) e flavonoides totais (FLAT).

\begin{tabular}{|c|c|c|c|c|c|c|c|c|c|c|}
\hline \multirow{2}{*}{ Source } & \multicolumn{5}{|c|}{ CFT } & \multicolumn{5}{|c|}{ FLAT } \\
\hline & DF & SS & MS & F calc. & $p$ & DF & SS & MS & F calc. & $p$ \\
\hline Falta de ajuste & 5 & 5,1849 & 1,0370 & 3,6216 & 0,1182 & 5 & 0,0198 & 0,0039 & 2,7393 & 0,1752 \\
\hline Erro puro & 4 & 1,1453 & 0,2863 & & & 4 & 0,0058 & 0,0014 & & \\
\hline Erro total & 9 & 6,3302 & & & & 9 & 0,0256 & & & \\
\hline F tabelado & & & & & & & & & & \\
\hline
\end{tabular}




\subsubsection{Superfície de resposta e análise dos efeitos principais}

Observou-se, a partir dos dados de resposta, que, dentro da região experimental planejada, houve uma variação de 50,24\% no teor de CFT extraídos e de 58,79\% no teor de FLAT extraídos, indicando que o conjunto de variáveis envolvidas no planejamento exerce influência sobre a eficiência do processo extrativo.

Os coeficientes de regressão para as respostas CFT e FLAT, e sua significância estatística estão sumarizados na Tabela 4, e as influências de cada uma das variáveis e de suas interações para as respostas CFT e FLAT são apresentadas em diagramas de Pareto (Figura 3).

Tabela 4. Coeficientes de regressão do modelo para as variáveis resposta, compostos fenólicos totais (CFT) e flavonoides totais (FLAT).

\begin{tabular}{|c|c|c|}
\hline \multirow{2}{*}{ Fatores } & \multicolumn{2}{|c|}{ Coeficientes de regressão } \\
\hline & CFT & FLAT \\
\hline Intercepto & $24,1393^{\mathrm{A}}$ & $0,9600^{\mathrm{A}}$ \\
\hline \multicolumn{3}{|l|}{ Linear } \\
\hline Temperatura & $2,7072^{\mathrm{A}}$ & $0,0593^{\text {В }}$ \\
\hline Etanol & $-2,5877^{\mathrm{A}}$ & $-0,0632^{\text {B }}$ \\
\hline Tempo & $1,7369^{\mathrm{A}}$ & $0,0549^{\mathrm{B}}$ \\
\hline \multicolumn{3}{|l|}{ Quadrático } \\
\hline Temperatura & $1,0335^{\mathrm{B}}$ & $0,0356^{\mathrm{C}}$ \\
\hline Etanol & $-1,4738^{\mathrm{A}}$ & $-0,1409^{\mathrm{A}}$ \\
\hline Tempo & $0,0503^{\mathrm{NS}}$ & $-0,0411^{\mathrm{C}}$ \\
\hline \multicolumn{3}{|l|}{ Interação } \\
\hline Temperatura $\times$ Etanol & 0,1043 NS & $-0,0355^{\mathrm{NS}}$ \\
\hline Temperatura $\times$ Tempo & $0,3825^{\mathrm{NS}}$ & $0,0405^{\mathrm{C}}$ \\
\hline Etanol $\times$ Tempo & $0,3130 \mathrm{NS}$ & $-0,0224 \mathrm{NS}$ \\
\hline $\mathrm{R}^{2}$ & $97,84 \%$ & $95,09 \%$ \\
\hline Erro puro & 0,2863 & 0,0014 \\
\hline
\end{tabular}

Significância estatística para: ${ }^{\mathrm{A}} p<0,001 ;{ }^{\mathrm{B}} p<0,01 ;{ }^{\mathrm{C}} p<0,05 ;{ }^{\text {NS }}$ Não significativo.
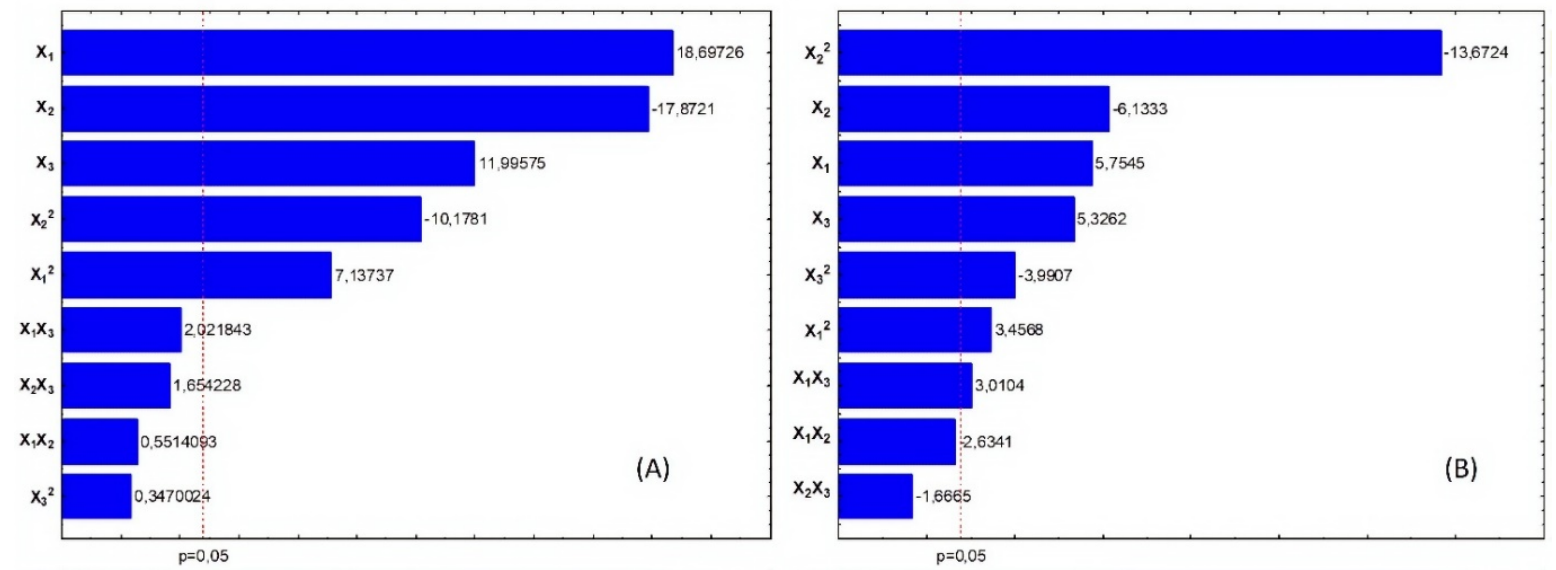

Figura 3. Influência das variáveis $\mathrm{X}_{1}\left(\mathrm{~T}^{\circ} \mathrm{C}\right), \mathrm{X}_{2}(\mathrm{EtOH} \%)$ e $\mathrm{X}_{3}(\mathrm{t}$ min) e de suas interações sobre as variáveis de resposta CFT (A) e FLAT (B).

Para a resposta CFT, a Figura 3A mostra que os efeitos lineares para temperatura e tempo exercem influência positiva no processo extrativo, isto é, o aumento em grandeza dessas variáveis reflete em um aumento na eficiência na extração de CFT. Este comportamento foi oposto ao observado para a variável concentração de etanol, que apresentou uma forte influência linear negativa sobre o processo, que pode ser corroborada pela relevante influência quadrática negativa apresentada para esta variável, significando que as concentrações medianas de etanol proporcionam maior eficiência na extração de CFT do que as concentrações extremas, principalmente as concentrações que tendem para as mais elevadas. Isso pode ser 
facilmente observado nas curvaturas das superfícies de resposta para essa variável, tanto em relação à variável tempo quanto em relação à variável temperatura de extração (Figura 4).

Como ilustrado na Figura 3B, a variável concentração de etanol apresentou uma forte influência quadrática negativa sobre a resposta FLAT, o que pode ser observado pela pronunciada curvatura nas superfícies de resposta para esta variável, tanto em relação à variável tempo quanto em relação à variável temperatura de extração, significando que as concentrações medianas de etanol proporcionam maior eficiência na extração de FLAT e que esta eficiência diminui drasticamente quando as concentrações se afastam dos valores medianos em direção aos valores extremos menores ou maiores. As variáveis tempo e temperatura de extração apresentaram influência linear positiva moderada sobre o processo extrativo, a qual pode ser percebida pela sutil inclinação apresentada por estas duas variáveis em relação à variável concentração de etanol nos respectivos gráficos de superfície. É importante destacar que, mesmo que fraca, existe influência da interação linear entre as variáveis tempo e temperatura de extração, e, ao se observar o gráfico de superfície para estas duas variáveis, percebe-se também que o efeito quadrático negativo do tempo de extração exerce maior influência sobre o processo do que a interação linear entre estas duas variáveis, sugerindo que, em processos extrativos mais demorados, é provável que ocorra uma diminuição da resposta FLAT (Figura 4). A literatura científica reporta comportamentos semelhantes ao observado neste estudo para as variáveis tempo de extração e concentração de etanol, relatando que a eficiência nos processamentos extrativos responde moderadamente ao tempo e aumenta com o emprego de soluções hidroalcoólicas de concentrações medianas, em torno de $60 \%$, e em temperaturas abaixo de $70{ }^{\circ} \mathrm{C}$ (Ahmed et al., 2011; Spagolla et al., 2009; Aguiar et al., 2007; Lapornik et al., 2005). 

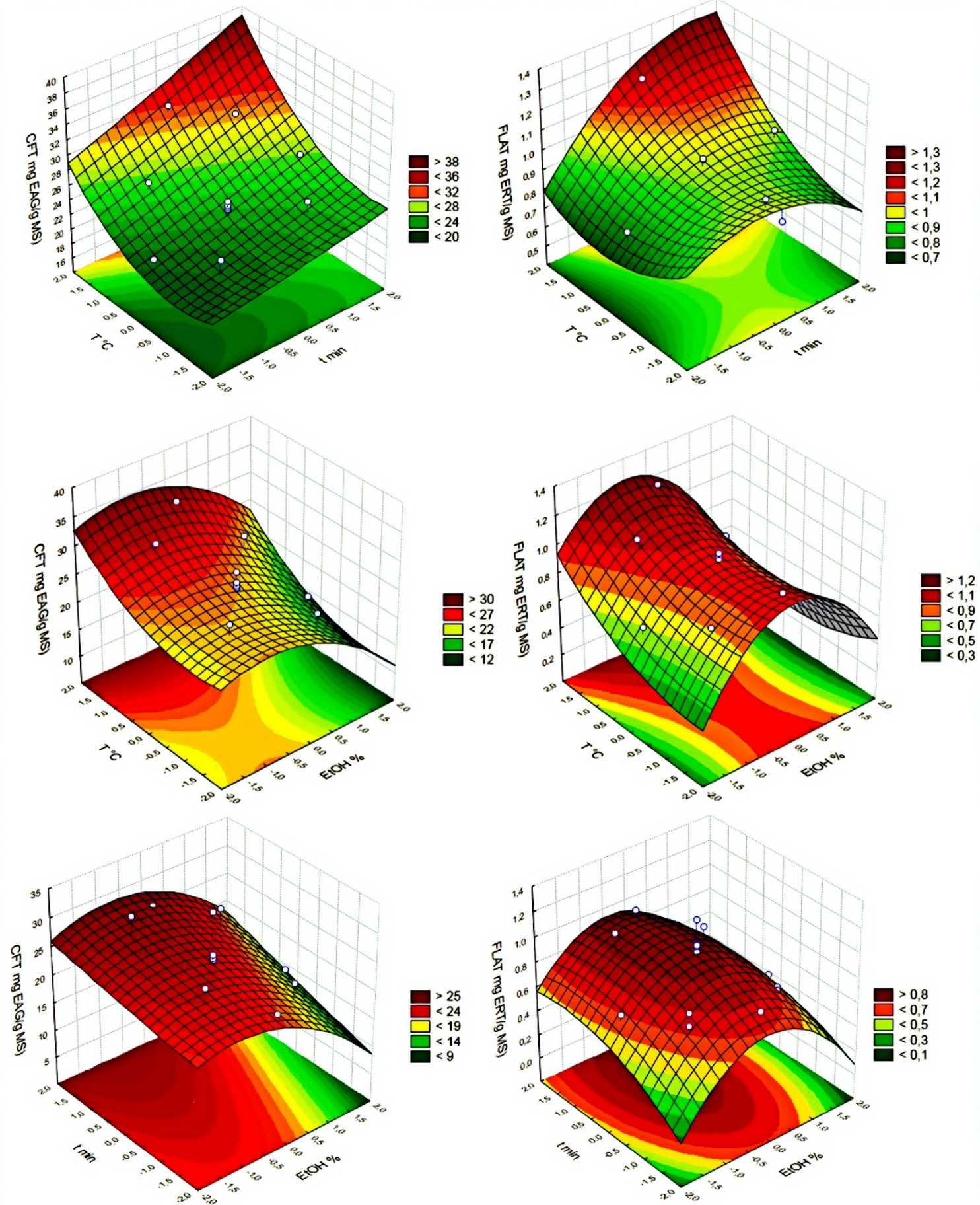

Figura 4. Superfície de resposta e contorno para as respostas CFT (esquerda) e FLAT (direita), em função das variáveis $\mathrm{X}_{1}\left(\mathrm{~T}^{\circ} \mathrm{C}\right), \mathrm{X}_{2}(\mathrm{EtOH} \%)$ e $\mathrm{X}_{3}(\mathrm{t} \mathrm{min})$.

\subsubsection{Otimização do processo de extração simultânea de compostos fenólicos}

O modelo estatístico delineado e testado neste estudo forneceu as equações de predição 2 e 3 para as respostas CFT e FLAT, respectivamente.

$$
\begin{aligned}
& C F T=24,14+2,71 X_{1}-2,59 X_{2}+1,74 X_{3}+1,03 X_{1}^{2}-1,47 X_{2}^{2}+0,05 X_{3}^{2}+0,10 X_{1} X_{2}+0,38 X_{1} X_{3}+0,31 X_{2} X_{3} \\
& F L A T=0,96+0,06 X_{1}-0,06 X_{2}+0,05 X_{3}+0,04 X_{1}^{2}-0,14 X_{2}^{2}-0,04 X_{3}^{2}-0,03 X_{1} X_{2}+0,04 X_{1} X_{3}-0,02 X_{2} X_{3}
\end{aligned}
$$


A condição ótima para a extração simultânea de CFT e FLAT foi predita pela função de desejabilidade global do modelo, assumindo os valores teóricos descritos na Tabela 5, com os parâmetros de desejabilidade assumidos listados na Tabela 6.

Tabela 5. Condições otimizadas para a extração simultânea de CFT e FLAT.

\begin{tabular}{|c|c|}
\hline Condições otimizadas $^{\mathbf{A}}$ & Variáveis \\
\hline Temperatura $\left({ }^{\circ} \mathrm{C}\right)$ & 64,18 \\
\hline Etanol (\%) & 60,6 \\
\hline Tempo (min) & 71,93 \\
\hline Valores preditos ${ }^{B}$ & Respostas \\
\hline CFT & $35,265 \pm 2,22$ \\
\hline FLAT & $1,210 \pm 0,16$ \\
\hline \multicolumn{2}{|c|}{ Valores Observados $^{\mathrm{C}}$} \\
\hline CFT & $33,234 \pm 1,66$ \\
\hline FLAT & $1,216 \pm 0,008$ \\
\hline
\end{tabular}

${ }^{\mathrm{A}}$ Desejabilidade global; ${ }^{\mathrm{B}}$ Média $\pm \mathrm{IC}=95 \% ;{ }^{\mathrm{C}}$ Média $\pm \mathrm{DP}(\mathrm{n}=3)$.

Tabela 6. Parâmetros de desejabilidade assumidos na otimização de compostos fenólicos totais (CFT) e flavonoides totais (FLAT).

\begin{tabular}{cccccc}
\hline \multirow{2}{*}{ Variáveis de resposta } & \multicolumn{5}{c}{ Parâmetros assumidos na otimização } \\
\cline { 2 - 5 } & Baixo & Médio & Alto & s & t \\
\hline CFT mg EAG/g MS & $16,289(0)$ & $24,357(0)$ & $32,424(1)$ & 1 & 1 \\
FLAT mg EAG/g MS & $0,4970(0)$ & $0,852(0)$ & $1,206(1)$ & 1 & 1 \\
\hline
\end{tabular}

(0): Valores indesejáveis; (1): Valores aceitáveis para a função desejabilidade; s e t: Expoentes da função.

Os valores observados experimentalmente estão dentro de um intervalo de confiança médio de $95 \%$ do valor predito para as respostas CFT e FLAT, resultados que confirmam o modelo como preditivo para extração simultânea dos compostos fenólicos presentes na casca dos frutos de $H$. courbaril L. dentro da região experimental explorada neste estudo.

Estudos usando a metodologia de superfície de resposta podem fornecer novas perspectivas positivas no aproveitamento de produtos naturais de origem vegetal e de seus resíduos, contribuindo no desenvolvimento de tecnologias que podem ser transferidas para a sociedade, tanto para processos em pequena escala quanto para processos industriais.

\section{Conclusão}

O modelo polinomial de segunda ordem foi capaz de descrever satisfatoriamente os dados experimentais obtidos para as respostas de compostos fenólicos totais (CFT) e flavonoides totais (FLAT), as quais mostraram comportamentos distintos na região experimental delineada para este estudo, em que se observou forte influência linear positiva das variáveis tempo e temperatura de extração e também uma considerável influência quadrática negativa da variável concentração de etanol sobre a resposta CFT. Por outro lado, a resposta FLAT foi fortemente afetada pela influência quadrática negativa da variável concentração de etanol, indicando concentrações em torno de $60 \%$ como as mais eficientes. A concentração de etanol pode ser considerada como o fator mais relevante neste processo extrativo simultâneo de compostos fenólicos da casca dos frutos de Hymenaea courbaril L., uma vez que, a partir de 50 minutos, a influência da variável tempo não aumentou significativamente a resposta CFT e pode diminuir a resposta FLAT, assim como temperaturas acima de $70{ }^{\circ} \mathrm{C}$ podem favorecer a degradação destes compostos. 


\section{Referências}

Aguiar, C. L., Baptista, A. S., Alencar, S. M., Haddad, R., \& Eberlin, M. N. (2007). Analysis de isoflavonoids from Leguminous plant extracts by RPHPLC/DAD and electrospray ionization mass spectrometry. International Journal of Food Sciences and Nutrition, 58(2), 116-124. PMid:17469767. http://dx.doi.org/10.1080/09637480601149350

Ahmed, M., Akter, S., \& Eun, J. B. (2011). Optimization conditions for anthocyanin and phenolic content extraction form purple sweet potato using response surface methodology. International Journal of Food Sciences and Nutrition, 62(1), 91-96. PMid:20858156. http://dx.doi.org/10.3109/09637486.2010.511167

Aquino, A. C. M. S., Móes, R. S.; Leão, K. M. M.; Figueiredo, A. V. D.; Castro, A. A. (2010). Avaliação físico-química e aceitação sensorial de biscoitos tipo cookies elaborados com farinha de resíduos de acerola. Revista do Instituto Adolfo Lutz, 69(3), 379-386.

Campos, M. A. A., \& Uchida, T. (2002). Influência do sombreamento no crescimento de mudas de três espécies amazônicas. Pesquisa Agropecuária Brasileira, 37(3), 281-288. http://dx.doi.org/10.1590/S0100-204X2002000300008

Cavalcanti, M., Selvam, M. M., Vieriea, R. R. M., Colombo, C. R., \& Queiroz, V. T. M. (2010, Outubro). Pesquisa e desenvolvimento de produtos usando resíduos de frutas regionais: Inovação e integração no mercado competitivo. Paper presented at the 30th Encontro Nacional de Engenharia de Produção: Maturidade e desafios da Engenharia de Produção: competitividade das empresas, condições de trabalho, meio ambiente (pp. 1-9). São Carlos, São Paulo: ABEPRO.

Dornas, W. C., Oliveira, T. T., Dores, R. R. G., \& Nagem, T. J. (2007). Flavonóides: potencial terapêutico no estresse oxidativo. Revista de Ciências Farmacêuticas Básica e Aplicada, 28(31), 241-249.

Escribano-Bailon, M. T., \& Santos-Buelga, C. (2003). Polyphenol extraction from foods. In C. Santos-Buelga \& G. Williamson (Eds.), Methods in polyphenol analysis (pp. 1-16). Cambridge: Royal Society of Chemistry.

Fernandes, H. P., Sinhorin, A. P., Shinhorin Junior, V. D. G., Silva, G. M. V., \& Carvalho, V. C. (2015). New glycosylated biscoumarins from Hymenaea coubaril L. seeds. Phytochemistry Letters, 13, 413-416.

http://dx.doi.org/10.1016/j.phytol.2015.07.016

Gironés-Vilaplana, A., Baenas, N., Villaño, D., Speisky, H., García-Viguera, C., \& Moreno, D. A. (2014). Evaluation of LatinAmerican fruits rich in phytochemicals with biological effects. Journal of Functional Foods, 7, 599-608. http://dx.doi.org/10.1016/j.jff.2013.12.025

Gordon, A., Jungfer, E., Silva, B. A., Maia, J. G. S., \& Marx, F. (2011). Phenolic constituents and antioxidant capacity of four underutilized fruits from the amazon region. Journal of Agricultural and Food Chemistry, 59(14), 7688-7699. PMid:21662239. http://dx.doi.org/10.1021/jf201039r

Infante, J., Selani, M. M., Toledo, N. M. V., Diniz, M. F. S., Alencar, S. M., \& Spoto, M. H. F. (2013). Atividade antioxidante de resíduos agroindustriais de frutas tropicais. Alimentos e Nutrição Araraquara, 24(1), 92.

Ishimoto, F. Y., Harada, A. I., Branco, I. G., Conceição, W. A. S., \& Coutinho, M. R. (2007). Aproveitamento alternativo da casca do Maracujá-Amarelo (Passiflora edulis f. var. flavicarpa Deg.) para produção de biscoitos. Ciências Exatas e Naturais, 9(2), 279-292.

Jerez, M., Pinelo, M., Sineiro, J., \& Núñez, M. J. (2006). Influence of extraction conditions on phenolic yields from pine bark: assessment of procyanidins polymerization degree by thiolysis. Food Chemistry, 94(3), 406-414.

http://dx.doi.org/10.1016/j.foodchem.2004.11.036

Khuri, A. I., \& Cornell, J. A. (1996). Response surfaces: designs and analyses (2a ed.) New York, NY: Marcel Dekker.

Lamaison, J. L. C., \& Carnet, A. (1990). Teneurs en principaux flavonoids des fleurs de Crataegeus monogyna Jacq et de Crataegeus laevigata (Poiret D. C) en fonction de la vegetation. Pharmaceutica Acta Helvetiae, (65), 315-320.

Lapornik, B., Prosek, M., \& Wondra, A. G. (2005). Comparison of extracts prepared from plant by-products using different solvents and extraction time. Journal of Food Engineering, 71(2), 214-222. http://dx.doi.org/10.1016/j.jfoodeng.2004.10.036

Lorenzi, H., \& Matos, F. J. A. (2002). Plantas medicinais no Brasil: nativas e exóticas cultivadas (512 p.). Nova Odessa, SP: Instituto Plantarum.

Machado, H., Nagem, T., Peters, V., Fonseca, C., \& Oliveira, T. (2008). Flavonóides e seu potencial terapêutico. Boletim do Centro de Biologia da Reproducao, 27(1/2), 33-39.

Mafart, P., \& Béliard, E. (1992). Génie industriel alimentaire: techniques séparatives (Vol. 2, 288 p.). Paris: Tec \& Doc Lavoisier.

Matos, N. M. S.; Silva, M. S.; Silva, S. M.; Moura, F. T., \& Alves, R. E. (2009). Alterações físicas e físico-químicas durante o desenvolvimento e maturação de jatobá (Hymenae stigonocarpa Mart.). In 12a Congresso Brasileiro de Fisiologia Vegetal (pp. 354, Livro de Resumos). Fortaleza: Sociedade Brasileira de Fisiologia Vegetal.

Montgomery, D. C., \& Runger, G. C. (2003). Applied statistics and probability for engineers (3. ed.). New York, NY: Willey.

Neves, M. C. A., Neves, P. C. A., Zanini Junior, J. C., Medeiros, Y. S., Yunes, R. A. \& Calixto, J. B. (1993). Analgesic and antiinflammatory activities of the crude hydroalcoholic extract obtained from the bark of Hymenaea martiana. Phytotherapy Research, 7(5), 356-362. http://dx.doi.org/10.1002/ptr.2650070507

Nimalaratne, C., \& Wu, J. (2015). Hen egg as antioxidante food commodity: a review. Nutrients, 7(10), 8274-8293.

PMid:26404361. http://dx.doi.org/10.3390/nu7105394 
Uso da metodologia de superfície de resposta na otimização da extração de compostos fenólicos da casca dos frutos de Hymenaea courbaril L. (Jatobá)

Silva, R. W. V. et al.

Nogueira, R. T., Shepherd, G. J., Laverde Junior, A., Marsaioli, A. J., \& Imamura, P. M. (2001). Clerodane-type diterpenes from the seed pods of Hymenaea courbaril var. stilbocarpa. Phytochemistry, 58(8), 1153-1157. PMid:11738399.

http://dx.doi.org/10.1016/S0031-9422(01)00303-X

Patil, B. S., Jayaprakasha, G. K., Chidambara Murthy, K. N., \& Vikram, A. (2009). Bioactive compounds: historical perspectives, opportunities and challengers. Journal of Agricultural and Food Chemistry, 57(18), 8142-8160. PMid:19719126. http://dx.doi.org/10.1021/jf9000132

Singleton, V. L., \& Rossi, J. A. J. (1965). Colorimetry of total phenolics with phosphomolybdic-phosphotungstic acid reagents. American Journal of Enology and Viticulture, (16), 144-158.

Spagolla, L. C., Santos, M. M., Passos, L. M. L., \& Aguiar, C. L. (2009). Extração alcoólica de fenólicos e flavonóides totais de mirtilo "Rabbiteye" (Vaccinium ashei) e sua atividade antioxidante. Journal of Basic and Applied Pharmaceutical Sciences, 30(2), 187-190.

Uchoa, A. M. A., Costa, J. M. C., Maia, G. A., Silva, E. M. C., Carvalho, A. F. F. U., \& Meira, T. R. (2008). Parâmetros físicoquímicos, teor de fibra bruta e alimentar de pós alimentícios obtidos de resíduos de frutas tropicais. Segurança Alimentar e Nutricional, 15(2), 58-65. http://dx.doi.org/10.20396/san.v15i2.1817

Financiamento: Nenhum. 